\title{
Microstructure of TiN Coatings by EBSD Techniques
}

\author{
Bong-Yong Jeong, S.Yue, R.Gauvin and R.A.L. Drew
}

Department of Mining, Metals and Materials Engineering, McGill University, 3610 University St. Montreal, Quebec, H3A 2B2, Canada

Titanium nitride, TiN, has superior characteristics such as good wear and corrosion resistance, a low friction coefficient, good thermal and electrical conductivity and biocompatibilty. Therefore, TiN coatings have been widely used in the field of cutting tools, diffusion barrier coatings in integrated circuit fabrication and turbine blade for jet plane engine and power plant.

In order to evaluate the microstructure of TiN coatings, many techniques are used, such as scanning electron microscopy (SEM), atomic force microscopy (AFM), transmission electron microscopy (TEM), X-ray photoelectron spectroscopy (XPS), Auger electron spectroscopy (AES) and so on.

The electron back scatter diffraction (EBSD) technique is a newly established tool for quantitative characterization of microstructures $[1,2]$. This has recently been extended to investigate thin film structures and oxide scale layers [3,4]. The automatic processing and analysis of EBSD patterns have enabled characterization of the local crystallography and grain boundary structure. This technique is also known as orientation image microscopy (OIM), and it allows us to analyze not only crystal orientation but also microstructure [5]. In the OIM, the image quality (IQ) describes the quality of the diffraction pattern obtained at a particular point in the measurement grid. In the IQ map, the contrast arises due to local differences in the orientation of the crystal lattice. Thus, the IQ map represents not only a qualitative measure of the imperfection of crystal lattice, but also indicates the presence of grain boundaries as well as subgrain boundaries.

In this work, the substrate material used was a Ni superalloy and a $6 \mu \mathrm{m}$ thick TiN coating was deposited by a Physical Vapor Deposition (PVD) method. After mounting, the specimens were mechanically polished using $\mathrm{SiC}$ paper, $1 \mu \mathrm{m}$ diamond paste, and $0.05 \mu \mathrm{m}$ colloidal silica in succession. The EBSD data was obtained using a Philips XL-30 FEG with TSL orientation imaging system.

Figure 1 illustrates the IQ map of the TiN layer. It is shows the very small grains in the coatings layer with each of the gray contrasts level corresponding to a different orientation. The dark lines in this figure show the high angle grain boundaries. Figure 2 shows the crystal orientation map for the same area of Fig. 1, in normal direction. The figure clearly shows the microstructure of the TiN grains as well as that of the subgrain boundaries. TiN grains are obviously visible as small columnar grains between $0.15 \mu \mathrm{m}$ and 1.5 $\mu \mathrm{m}$. In Fig. 2, the black spots are results of the beam drift phenomenon during EBSD map scanning. Figures 3 and 4 show the color key used to produce the crystal orientation map (COM) and distribution of the TiN grain size, respectively. We can obtain the information of the crystal orientation and the grain size of the TiN coatings.

It is clear from the EBSD results that it can be used to determine the grain size and the growth characteristics of TiN coatings. Moreover, IQ mapping with the orientation information has a strong potential to reveal more microstructural characteristics of hard coatings. This will improve the understanding of the coating mechanisms and properties.

References

[1] A.J.Wilkinson and P.B.Hirsch, Micron. 28 (1997) 279.

[2] F.J.Humphreys, J.Microsc. 195, (1999) 170.

[3] D.P.Burke and R.L.Higginon, Scripta Mater. 42, (2000) 277.

[4] Bae-Kyun Kim and J.A.Szpunar, Scripta Mater. 44, (2001) 2605. 
[5] D.J.Dingley and D.P.Field, Mater. Sci. Technol., 13, (1997) 69.

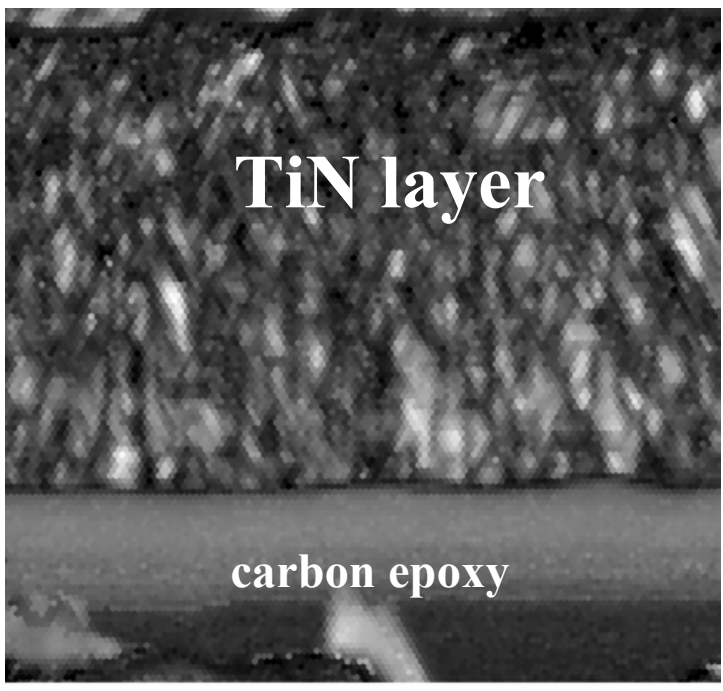

$2.50 \mu \mathrm{m}=25$ steps $I Q 9.1 \ldots 79$

Fig. 1. IQ map of TiN coatings on the Ti alloy, where the black scale level in the image corresponds to the quality of the EBSD pattern at that point.

\section{Gray Scale Map Type: snone $>$}

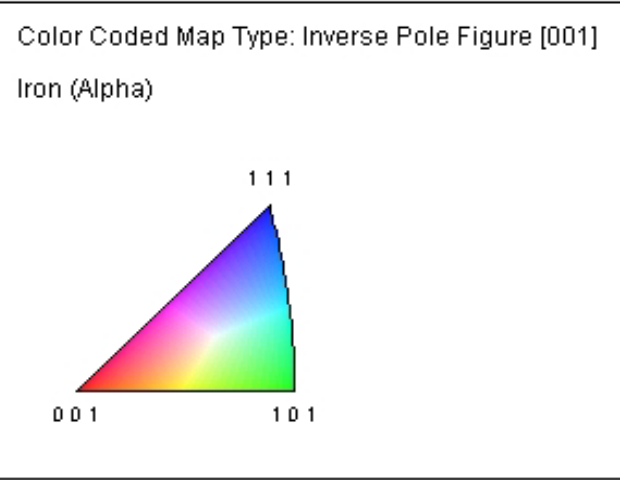

Boundaries: «none?

Fig. 3. Color key used to produce the COM shown in figure 3 , where the color is given by the direction of the sample normal direction in the inverse pole figure.

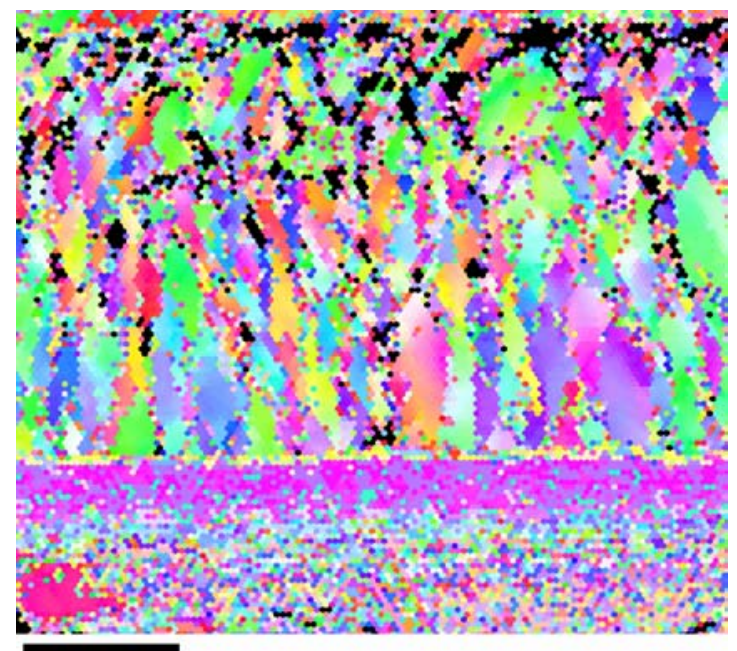

$2.50 \mu \mathrm{m}=25$ steps IPF [001]

\section{Normal direction}

Fig. 2. Crystal orientation map (COM) for the area shown in figure 1 , which is showing regions with a similar crystal orientation in the same color.

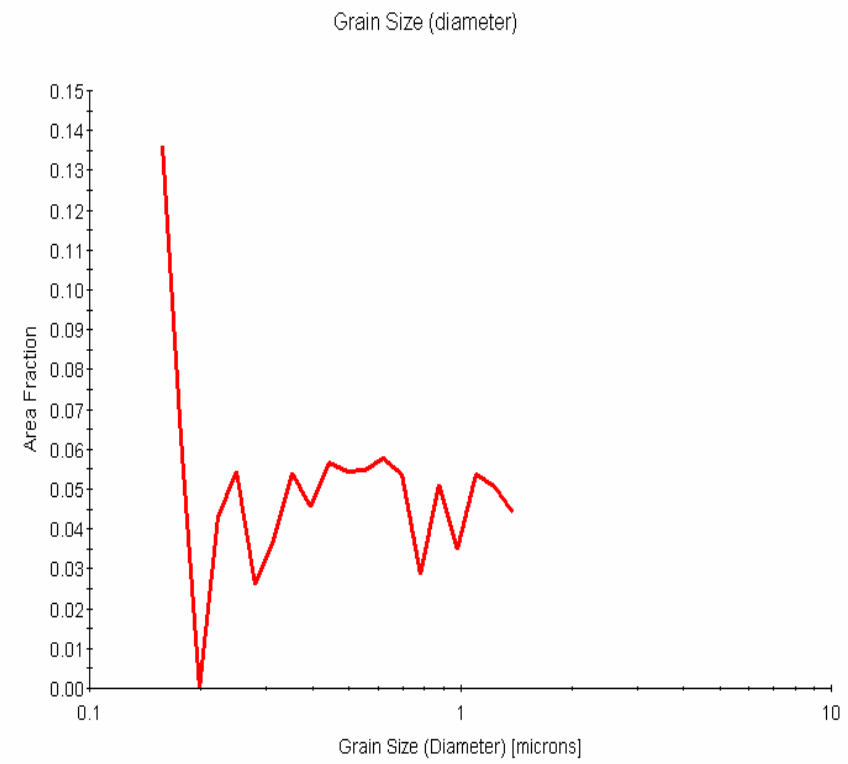

Fig. 4. Distribution chart of the TiN grain size. 